nation of these bones. I would only observe, that before rejecting the mechanical explanation, we must thoroughly in vestigate the particular case : for the direction of the greatest muscular strain is as effectual a cause of bending, as the direction of the least bony resistarce; and I can easily conceive the muscles of the calf to have determined the direction of the curve here.

The feet need not occupy us long. All the bones have been preserved except a few phalanges which have melted away under the protracted maceration. The tarsal bones are many of them distorted, though not to such an extent as to throw any real difficulties in the way of their proper arrangement. And they are all small and ill-developed, contrasting strongly with the bones of the hands. But it is not surprising that they have yielded to the constrained posture in which they were placed, considering that what we call bones by courtesy, are really nothing more than fatty matter, contained within a thick case of periosteum.

\section{THE PATHOLOGY, DIAGNOSIS, AND TREATMENT OF CARDIAC DISEASES.}

By W. O. M.nkham, M.D., F.R.C.P., Physician to St. Mary's Hospital, London.

III.-Causes of Pericarditis.

InFLAmMation of the pericardium is in no case to be regarded as an idiopathic inflammation. It may in almost every in. stance be traced either to local injury, or to some morbid condition of the system generally. Pericarditis, as the result of direct local irritation, has many different exciting causes. It may be produced by traumatic injuries, by blows and wounds. Tubercle or cancer deposited upon the serous surface of the membrane, or spreading into the sac from the parts around, will occasion it; so also may abscess of the liver, of the medias. tina, and of the neighbouring parts, hydatids of the liver, ulceration of the œsophagus and of the stomach, etc., by opening into the sac, and there discharging their contents. Pleurisy, pneumonia, and peritonitis-all these, and similar local sources of irritation, may likewise excite inflammation in the membrane.*

Tubercle and cancer very rarely, if ever, arise primitively in the pericardium. These diseases almost invariably preexist in some other parts or organs of the body, before they appear here. Cancer generally spreads into the sac from the mediastinum, or some neighbouring organ affected with the morbid growth.

Pericarditis is more especially known to us as it appears in association with general disorders-with rheumatic fever, kidney diseases, tuberculosis, pyæmia, and the acute exanthemata. In such case, it is manifestly the local manifestation of a general affection of the body-the product, perhaps, of some morbid condition of the blood. But, though we have in these diseases evidences enough to satisfy us of the existence of some deleterious agent in the system, we are still quite in the dark as to the causes which determine the disease to fix itself especially in the pericardium.

If we reflect upon the catalogue of diseases which authors record as causes of pericarditis, we shall perceive that what are frequently written down as causes are, in reality, merely coexisting manifestations of the self-same disorder as that which provoked the pericarditis. In this sense, acute rheumatism cannot properly be called the cause of pericarditis. Both the affections are the resultants of a morbid state of the blood, and, as it would seem, of the same morbid state. What may be truly said of their association and relationship is this-that the morbid element of rheumatism more frequently displays its presence in the joints than it does in the pericardium; and more frequently at an early than at a late period of life. This becomes still more clear, if we call to mind the fact, that the

* I once sarr a case of pericarditis, which resulted from ulceration of the cesophagus. The ulcer in its progress opened at last into the pericardium, This is the only case of pneuno-pericardium to pass into the pericardium. air, of course, came from the stomach in this inich I have ever seen. The upon the stomach, its contents were readily forced, and seen to pass, up the csophagus, and so through the ulcerated operced, and seen to pass, up pericardial inflammation does occasionally precede the arthritic affection in rheumatic pericarditis.*

Dr. T. K. Chambers, in his Decennium Pathologicum, gives us a list of one hundred and thirty-five cases of diseases in which recent signs of pericardial inflammation were found after death. These diseases are, as I have observed, ordinarily termed causes of pericarditis, but must be rather designated as coexisting disorders.

Ditto, with diseased heart and kidneys $\quad . \quad \cdot \quad 1$

Uræmia from diseased kidneys, either alone, or with diseased heart

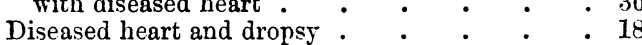

Pyæmia

Pneumonia

Pleurisy

Vomicæ in the lungs . . . . . . . .

Erysipelas

Typhus

Other causes

A glance at this table shows us, as Dr. Chambers remarks, how decidedly the constitutional predominate over the (socalled) local causes of pericarditis. Dr. Ormerod, for the better comprehension of these so-called causes of pericarditis, has reduced them all under two heads, viz.: 1. Rheumatic pericarditis; 2. Non-rheumatic pericarditis. This division of the inflammation will be found of practical utility. A study of the clinical history of pericarditis shows us that there are marked points of distinction to be observed in its nature, which enable us readily to class the disease under one or other of the above heads. These distinctive signs give us a clue to the nature of the disease, showing how its characters vary in different cases, and consequently how varied, and even opposite, its proper treatment must be under such circumstances. I shall, therefore, point out the distinctive characters which appertain to the rheumatic and to the non-rheumatic kinds of pericarditis.

Rheumatic pericarditis occurs at a comparatively early period of life. The average age of sixty-one of Dr. Ormerod's patients was twenty-one. The subjects of the disease are, for the most part, of (apparently) healthy constitution. As regards sex, women appear as often as-perhaps somewhat more often thanmen, to be affected by it. The symptoms of the rheumatic pericarditis are prominent and well marked. The disease, except in rare instances, is associated with, and, in fact, preceded by rheumatic affections of the joints, which are of so striking a character that they cannot fail to excite the attention of the physician. The local pericardial inflammation, moreover, frequently reacts with violence on the system ; and in such case, when death ensues, it is to the immediate consequences of the pericarditis-to the action of the inflammation on the heart it self-not to the ordinary effects of rheumatism, that the fatal result is ascribable.

The heart is overwhelmed and paralysed by the local action of the inflammation upon it. But though the symptoms of rheumatic pericarditis are usually severe, the disease, when uncomplicated with other cardiac diseases and pulmonary affections, rarely destroys life by its direct and immediate effects. A first attack of rheumatic pericarditis is, indeed, very rarely fatal.

Many observations lead me to suspect that there is an hereditary tendency, or family predisposition, to rheumatic pericarditis in the young; though, for obvious reasons, it is very difficult to arrive at any positive conclusions on this point. I have, for instance, remarked the existence of cardiac disease in the children of parents who have themselves suffered from rheumatism and heart disease. The following are examples of the fact which have fallen under my notice :-

In one family, the father and two children had disease of the heart. The father had suffered from several severe rheumatic attacks before the birth of these children, and there is now a constant cardiac bruit audible over his heart. In the children, about 14 and 15 years of age, the heart is so impaired as to produce the severest symptoms of cardiac disease. One seems to have once suffered from pains in the ankles; but, as far as I could learn, the other never had suffered from any external symptom of rheumatism. Her first complaints of illness were short breath and dropsical swellings, the products of the cardiac disease.

A case of this kind is related by Dr. Graves, vol. ii, p. 159, "Clin. 
In another family, the father had for years been a martyr to rheumatism; and his child, about two years and a half old, had organic disease of the heart.

A child, 10 years old, had had rbeumatism, and when I saw her had mitral valvular disease. Her mother had been ailing twenty years, and a loud systolic aortic bruit was in her audible over the heart.

Moreover, it is not at all uncommon to see two, and even three, children in one family attacked with rheumatic pericarditis; but then it may be in some instances objected, that the children are all exposed to the same exciting infiuences of disease, and that, therefore, what has produced the affection in one, may also have excited it in the other.

Non.rheumatic pericarditis, on the other hand, occurs at a later period of life than the rhenmatic kind. Forty-two was the average age of persons affected by it in twenty-four cases reported by Dr. Ormerod. The subjects of the disease were, with a single exception, all of the male sex. The inflamma tion loes not so much attack the healthy, as those whose con stitutions are originally weak, or have been debilitated and depraved through indulgence in ill-habits of living. It is far more serious than rheumatic pericarditis in its immediate con sequences.

Dr. Ormerod informs us, as the results of his experience that $91 \cdot 6$ per cent, of his non-rheumatic cases were fatal; whilst of the rheumatic sort, 18 per cent only were fatal. The local symptoms, again, of non-rheumatic pericarditis are at times very obscure; they are often, indeed, so obscure as not to direct the observer by any local signs to the seat of the disease, and thus it not unfrequently happens that the existence of the pericarditis is first discovered only after death.* In correspondence with this fact, it may be observed that the local inflammation does not appear to exercise, by reaction (as happens in the case of rheumatic pericarditis), any particular influence over the patient's general condition, so as to appear in any direct way a cause of death. Moreover, the pathological changes found after death are not so considerable as those which result from rheumatic pericarditis, a circumstance which may explain, in some degree, the frequent absence of local symptoms during life in non-rheumatic pericarditis.

It is not, therefore, by the violence or the immediate effects of the local inflammation, that life is destroyed in cases of nonrheumatic pericarditis.t Death rather results from the general action of that particular disease which has, in fact, preceded, and has given rise to, the pericarditis. Thus, for example, when pericarditis appears in the course of kidney disease, it usually supervenes at an advanced period of the renal disorder; bu kidney disease (of the character here inferred) is, as we well know, for other reasons fatal in its consequence, and quite in dependently of its action upon the heart. The serous membranes, under the influence of uramic disease, readily take on inflammatory action; and, of all the membranes, the pleural and the pericardial appear to be most frequently subjected to it.

Thus, then, in non-rheumatic pericarditis, the local inflam mation plays a subordinate part. Its share, as a disturber of the system, is but small; so that it is, in fact, usually little more than a local sign (unimportant in its immediate effects) of a serious general disorder. In rheumatic pericarditis, on the other hand, the local inflammation must be considered, for the moment, as the all important fact; its consequences fall at once, and with violence, upon the heart; the inflammation is sthenic, and its products may be of such a nature as to ac directly on the heart, both impeding mechanically, and otherwise seriously deteriorating its powers. The general constitutional disorder with which it is associated is subordinate in importance to the local inflammation, which it has engendered.

The causes of non-rheumatic pericarditis, as may be inferred from what has been said at the head of this chapter, are of two kinds. They are local and constitutional. The chief local causes are: cancerous diseases, arising within the sac, or, as much more frequently happens, spreading into it from some of

* The extremely large fatality (as would appear from the statistics) of non-rheumatic pericarditis is, no doubt, more apparent than real; and fo this reason:-As non.rheumatic pericarditis is occasionally only disco ved alter denth, it is evident that it may have exisied in cases where recovery has taken place, having escaped observation during the progress of the particular disease with which it was associated. This fact is corextensive pericurdial adhesions, in cases where there is no history of the previous existence of any rheumatic affection.

+ In some rare cases, howerer, the amount of effusion is so great os mechanically to interfere with the heart's action. the parts around the pericardium; tuberculosis, pleuritis, and peritonitis, by acting as local sources of irritation; abscesses of neighbouring organs or parts; and hydatid cysts, by opening into and discharging their contents into the sac.

The chief constitutional causes, or, to speak more correctly, the diseases in connection with which the non-rheumatic pericarditis is observed, are diseases of the lidney, pleurisy, pneumonia, pyæmia, scarlatina, peritonitis, variola, etc.

Bright's disease of the kidney has been especially referred to by authors as a very frequent excitor of the inflammation. My own experience does not at all agree with this conclusion. Out of a creat number of cases of kidnev diseases which have come under my observation in the pathological department of the St. Mary's Hospital, I have met but with very few where there existed any recent signs of pericardial inflammation. The same results have been arrived at in Edinburgh, where kidney disease is very common.

Professor Bennett (Principles and Practice of Medicine) tells us, "That in none of his cases of pericarditis has there been a complication with Bright's disease."

Dr. Christison also says that pericarditis is rarely seen among the sequelæ of kidney disease.*

Happily, a knowledge of the actual relative proportion of the frequency of pericaraitis, in coincidence with kidney disease, is not of much practical importance to the physician; for the knowledge would in no manner advance his therapeutical powers over it. Pericarditis, when it does thus arise, comes on in the advanced stages of the kidney disease, and would, under such circumstances, claim no special attention as an object of treatment. In all the cases, indeed, of non-rheumatic pericarditis, of constitutional origin, the treatment has no direct reference to the pericardial inflammation; it is guided entirely by the nature of the constitutional disease with which it may happen to be associated. Herein it differs essentially from the treat. ment of rheumatic pericarditis.

Relation of Pericarditis to Rheumatic Fever. Statistics have as vet given us no sure information on this subject. M. Bouilland and Dr. Hope found pericarditis present in about one half of their cases of acute rheumatism ; but their experience does not at all coincide with the conclusions arrived at by later observers.

Dr. Taylor makes the number about one case of pericarditis in nine of rheumatic fever; Dr. Budd, about one in eight; Dr. Latham, about one in eight.

It is well to remark, however, that results on this subject, derived from the observation of cases which bave been taken indiscriminately, without regard to age or sex, are of very little value; for it is a fact that at certain periods of life the coincidence of the rheumatism and the pericarditis are much more common than they are at others. 'Thus, adult persons attacked by acute rheumatism are infinitely less liable to cardiac complications than the young are. After the age of thirty, pericardial and endocardial inflammations are comparatively rarely met with as the associates of rheumatism; whilst, on the other hand, before the age of twenty, and in early life, they are more frequently present than absent in connection with that disease.

Every physician must have frequently had occasion to corroborate the fact stated by Dr. Watson-a law he calls it-namely, that the younger the patient is who suffers acute rheumatism, the more likely will he be to have rheumatic carditis. Climate, also, it is probable, has something to do with the matter.t

\section{[To be continued.]}

* It is most probable that those writers who have made kidney diseases stand so high in the catalogue of causes of pericarditis, have set down as instances of the fact, all those cases in which pathological conditions of the pericardium have been found coexisting with kidney-diseases after death. But such calculations are manifestly incorrect. In many of these cases, it is certain that other diseases of the heart and of different organs of the body were present; and in many of these it would doubtless be equally correct (perhaps more correct) to call the cardiac disease the cause of the kidneydisease, as vice vers $\hat{t}$. Old pericardial adhesions coexisting with granular kidney, can in no way be called the consequences of the kidney-disease; they may have very possibly existed before the kidney-disease began. Of the cases of heart-disease recorded by Dr. Barclay (Med. Chir. Trans., vol. xxxy), I cannot find above two or three in which there is any apparently close relation between the kidney-disense and the pericardial nffection. But, on tho other hand, I find that in thirty-four of the minety-two cases there was marked killney-disease present without any pericardict action, and that there were found twenty-six cases of lcsions of the pericardium without any kin two hum ired and ninety two coses of Bright's disease.

+ Dr. Chevers Dr. Chevers states, that although articular (acute) rhenmatism is very affection is very rare. (Dublin Journal Med., $1 \% 2,1842$.) 